\title{
Pulmonary dirofilariasis: report of a human case
}

\author{
HOWARD K. LEONARDI, JANET D. LAPEY, AND F. HENRY ELLIS, JR
}

From the Lahey Clinic Foundation and New England Deaconess Hospital, Boston, Massachusetts, USA

Leonardi, H. K., Lapey, Janet D., and Ellis, F. H., Jr (1977). Thorax, 32, 612-615. Pulmonary dirofilariasis: report of a human case. Dirofilaria immitis (dog heartworm) principally infests canine hosts. However, human pulmonary dirofilariasis is being reported with increasing frequency, and the following case illustrates essential features of the disease. It typically presents as a solitary pulmonary nodule without symptoms and invariably requires pulmonary resection to differentiate it from primary or secondary malignancy.

Although pulmonary dirofilariasis was an unknown clinical entity in man before 1961, 46 cases have been reported since that time (Dashiell, 1961; Massachusetts General Hospital, 1974; Dayal and Neafie, 1975; Prioleau et al., 1976). The increasing number of reports makes a greater awareness of this disease particularly desirable among thoracic physicians and surgeons since it characteristically presents as a solitary pulmonary nodule. An additional well-documented illustrative case is presented here.

\section{Report of a case}

A 64-year-old resident of Brazil underwent pancreaticoduodenectomy at the Lahey Clinic Foundation for adenocarcinoma of the duodenum invading the head of the pancreas. The margins of the resection and all lymph nodes were free of tumour. The patient made an uneventful recovery and returned to Brazil where twice yearly follow-up assessments were carried out by his local physician.

Two and a half years after operation the patient returned for reassessment because of an abnormal chest radiograph. He had never smoked and was asymptomatic. Physical examination was unremarkable. A complete blood count revealed a moderate eosinophilia of $10 \%$. All other blood studies, including liver function tests, were within normal limits. A chest radiograph and tomograms showed a discrete $2 \mathrm{~cm}$ lesion in the right lower lung field without evidence of calcification (Figs 1 and 2). A liver scan gave negative results. A right thoracotomy was performed and a basal segmentectomy carried out. The pathological diagnosis was Dirofilaria immitis. The patient made an uneventful recovery and remains well 14 months later.

\section{PATHOLOGY}

The macroscopic specimen consisted of the basal segments of the right lower lobe and weighed $100 \mathrm{~g}$. An area of firm nodularity measured $1.0 \mathrm{~cm}$, was yellow-white, and was centrally friable. Barely visible in the centre was a minute, white, threadlike structure.

The microscopic sections showed a coiled worm $\overrightarrow{\overrightarrow{0}}$ measuring approximately $400 \mu \mathrm{m}$ located within a 3 pulmonary artery (Fig. 3). The worm had a multilayered cuticle with transverse striations $4 \mu \mathrm{m}$ apart and internal longitudinal cuticular ridges adjacent to broad lateral cords. The somatic 응 muscle was prominent, and an intestinal tract and $\times$ two uteri were visible. Ova were present, but no microfilariae were identified (Fig. 4).

The artery showed severe acute and chronic inflammation with intimal fibrosis and focal necrosis. Numerous plasma cells were present in the vessel $\stackrel{9}{\supset}$ wall. Smaller adjacent pulmonary vessels showed $\frac{D}{O}$ vasculitis with intimal fibrosis, and focal organising pneumonitis was present.

\section{Comment}

Dirofilaria immitis (the $\operatorname{dog}$ heartworm) is a nematode parasite whose principal hosts are dogs and other canines. It is presumed but unproved $\mathbb{D}$ that mosquitoes and possible other vectors, such as ?fleas, lice, and ticks, transmit the disease to man. A subcutaneous infection would precede micro- $\frac{\vec{D}}{\mathbb{D}}$ filaraemia and propagation of the parasite. How- $\frac{\Omega}{\Phi}$ ever, man's immunogenic milieu is apparently $\varrho$ unsuitable for completion of the parasite's life-? 


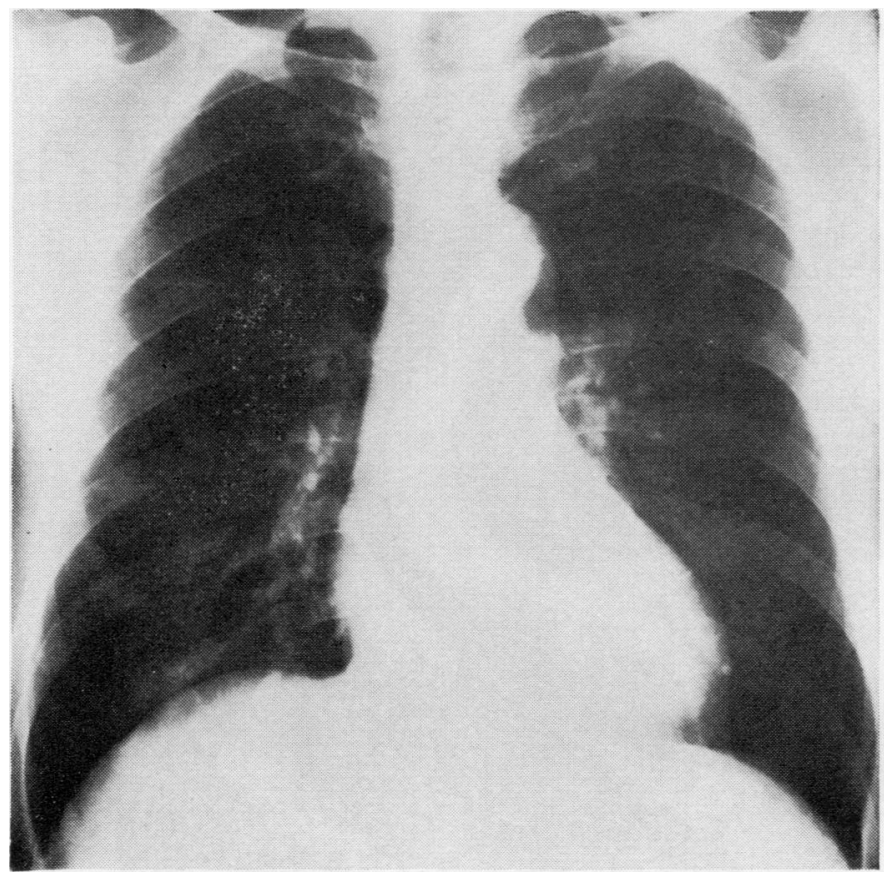

Fig. 1 Chest radiograph disclosing a $2 \mathrm{~cm}$ rounded shadow in the right lower lung field.

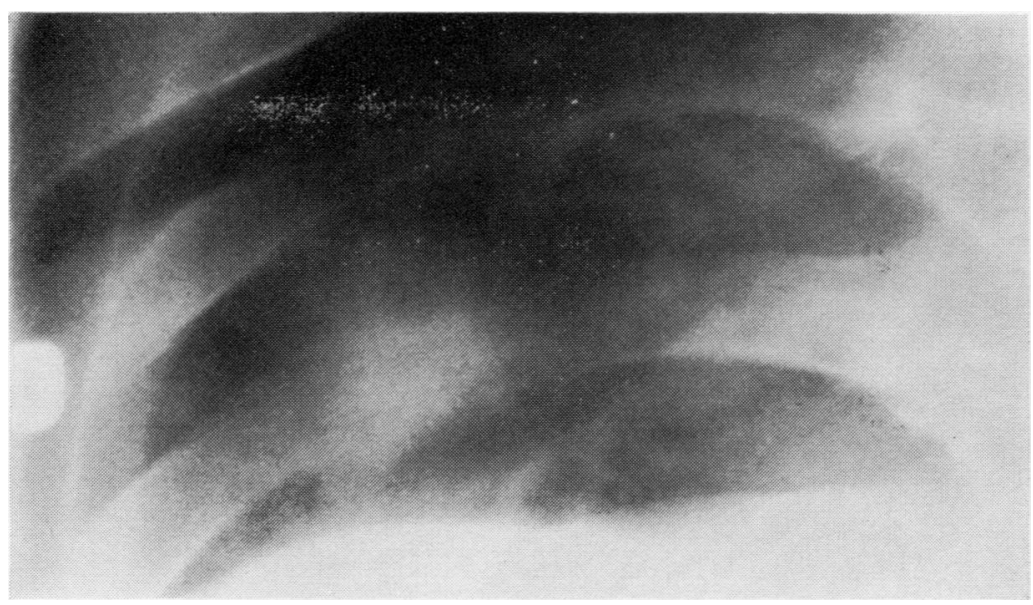

Fig. 2 Tomogram of the involved area reveals discrete margin and absence of calcification.

cycle and the dead worm lodges in a branch of the pulmonary artery, producing an ischaemic infarct with a characteristic surrounding reaction (Dayal and Neafie, 1975).

While most patients are asymptomatic, some reported cough, fever, and pleuritic pain several weeks before detection of the lesions. Fewer than $5 \%$ of the reported cases had the significant eosinophilia noted in this example. The radiological appearance is nonspecific, consisting of a well-circumscribed lesion varying in size from 1.5 to $6 \mathrm{~cm}$ without calcification or cavitation. There is no lobar predilection. Filarial antigens derived from Dirofilaria immitis have formed the basis for skin tests as well as haemagglutination and complement fixation tests. However, the yield of 


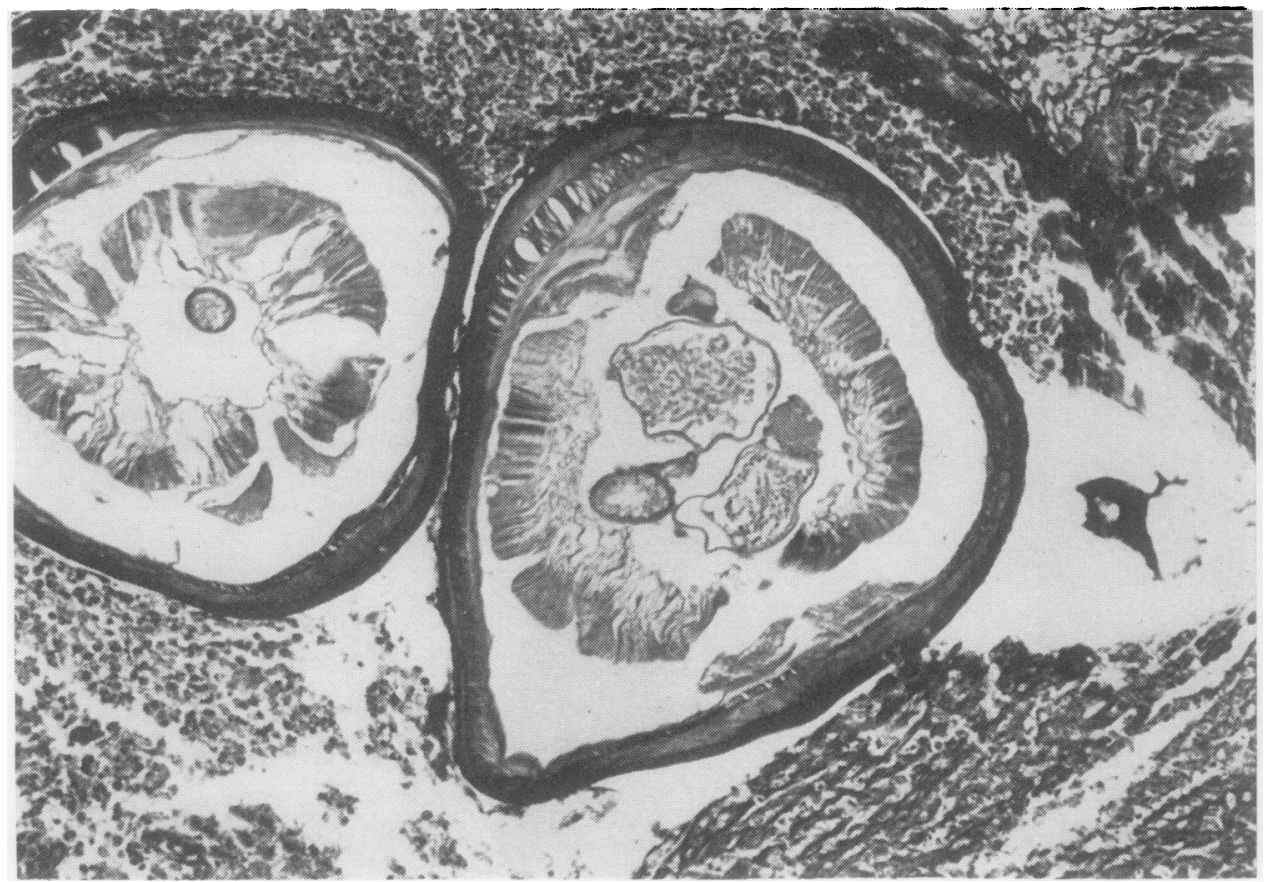

Fig. 3 Within the pulmonary artery is a coiled worm with a thick, multilayered cuticle, prominent somatic muscle, two uteri, and an intestinal tract (Verhoeff's stain $\times 77$ ).

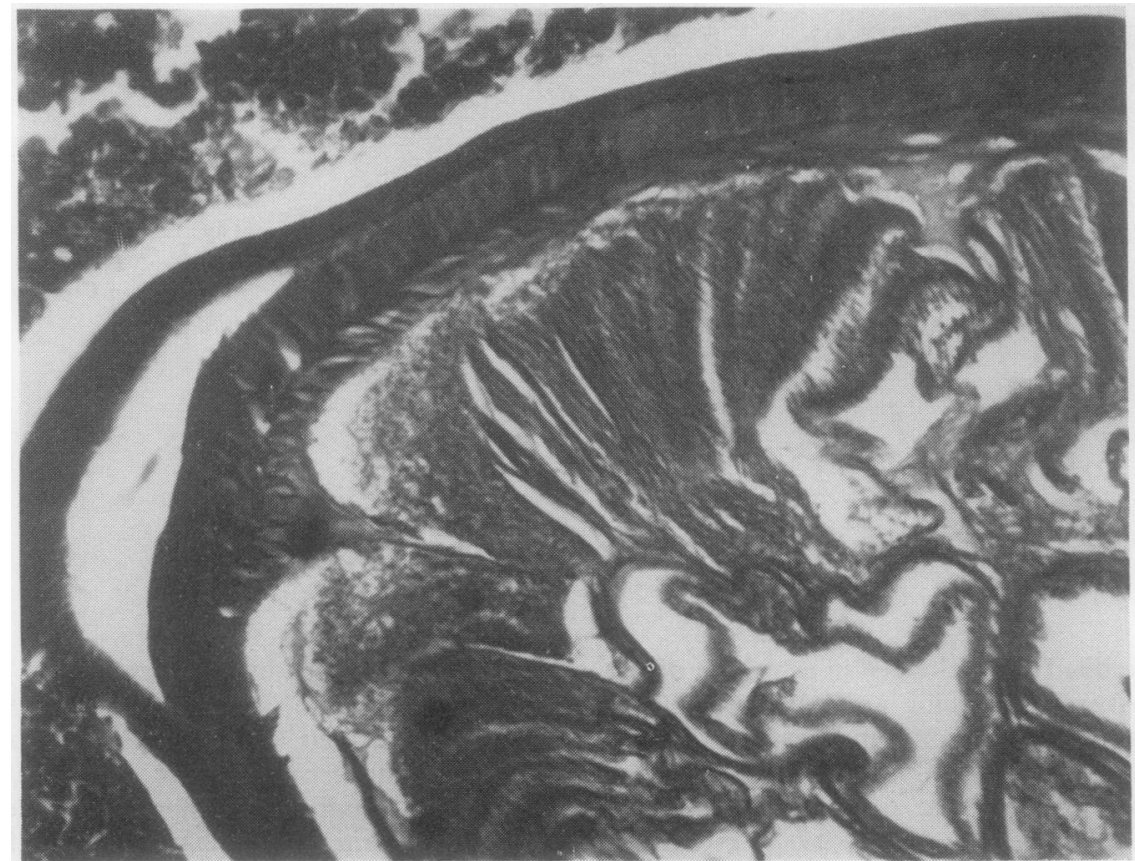

Fig. 4 Higher magnification demonstrates the transverse striations in the cuticle, the longitudinal internal cuticular ridge, and adjacent lateral cord (Verhoeff's stain $\times 400$ ). 
positive results is low in patients with proved pulmonary dirofilariasis (Massachusetts General Hospital, 1974).

The absence of a characteristic syndrome has made the clinical diagnosis of this increasingly reported disease elusive. In this example, as in all previously reported ones, the diagnosis followed pulmonary resection for suspected malignancy.

\section{References}

Dashiell, G. F. (1961). A case of dirofilariasis involving the lung. American Journal of Tropical Medicine and Hygiene, 10, 37-38.

Dayal, Y., and Neafie, R. C. (1975). Human pulmonary dirofilariasis: a case report and review of the literature. American Review of Respiratory Disease, 112, 437-443.

Massachusetts General Hospital (1974). Case records. Case 26-1974. New England Journal of Medicine, 291, 35-42.

Prioleau, W. H., Jr., Parker, E. F., Bradham, R. R., and Gregorie, H. B., Jr. (1976). Dirofilaria immitis (dog heartworm) as a pulmonary lesion in humans. Annals of Thoracic Surgery, 21, 382-385.

Requests for reprints to: Dr. F. H. Ellis, Jr, Department of Thoracic and Cardiovascular Surgery, Lahey Clinic Foundation, 605 Commonwcalth Avenue, Boston, MA, 02215, USA. 